\title{
Real-time data monitoring for ulcerative colitis: patient perception and qualitative analysis
}

\author{
Alissa Walsh ${ }^{1,2}$, Lawrence Matinii ${ }^{1}$, Christopher Hinds ${ }^{3}$, Vanashree Sexton ${ }^{4}$, Oliver Brain ${ }^{1}$, Satish Keshav ${ }^{1}$, \\ John Geddes ${ }^{4}$, Guy Goodwin ${ }^{4}$, Gary Collins ${ }^{5}$, Simon Travis ${ }^{1,2}$, Michele Peters ${ }^{6}$ \\ ${ }^{1}$ Translational Gastroenterology Unit, NIHR Oxford Biomedical Research Centre, Oxford University Hospitals NHS Foundation Trust, John \\ Radcliffe Hospital, Oxford; ${ }^{2}$ Linacre College, University of Oxford, Oxford; ${ }^{3}$ Big Data Institute, University of Oxford, Oxford; ${ }^{4}$ Department of \\ Psychiatry, University of Oxford, Oxford; ${ }^{5}$ Centre for Statistics in Medicine, University of Oxford, Oxford; ${ }^{6}$ Nuffield Department of Population \\ Health, University of Oxford, Oxford, UK
}

Background/Aims: TrueColours ulcerative colitis (TCUC) is a comprehensive web-based program that functions through email, providing direct links to questionnaires. Several similar programs are available, however patient perspectives are unexplored. Methods: A pilot study was conducted to determine feasibility, usability and patient perceptions of real-time data collection (daily symptoms, fortnightly quality of life, 3 monthly outcomes). TCUC was adapted from a web-based program for patients with relapsing-remitting bipolar disorder, using validated UC indices. A semi-structured interview was developed and audio-recorded face-to-face interviews were conducted after 6 months of interaction with TCUC. Transcripts were coded in NVivo11, a qualitative data analysis software package. An inductive approach and thematic analysis was conducted. Results: TCUC was piloted in 66 patients for 6 months. Qualitative analysis currently defies statistical appraisal beyond "data saturation," even if it has more influence on clinical practice than quantitative data. A total of 28 face-to-face interviews were conducted. Six core themes emerged: awareness, control, decision-making, reassurance, communication and burden of treatment. There was a transcending overarching theme of patient empowerment, which cut across all aspects of the TCUC experience. Conclusions: Patient perception of the impact of real-time data collection was extremely positive. Patients felt empowered as a product of the self-monitoring format of TCUC, which may be a way of improving self-management of UC whilst also decreasing the burden on the individual and healthcare services. (Intest Res 2019;17:365-374)

Key Words: Electronic data processing; Colitis, ulcerative; Perception

\section{INTRODUCTION}

Ulcerative colitis (UC) is a lifelong chronic IBD that follows an unpredictable relapsing and remitting course. ${ }^{1}$ When active, symptoms include urgent and frequent bloody diarrhea. The goal of treatment is to achieve remission, maintain remission and to achieve a good quality of life (QoL) for patients. QoL is often impaired in patients with UC. ${ }^{2,3}$ Education, employment,

Received January 4, 2019. Revised March 29, 2019. Accepted April 30, 2019. Correspondence to Alissa Walsh, Translational Gastroenterology Unit, John Radcliffe Hospital, Oxford OX3 9DU, UK. Tel: +44-7495920267, E-mail: alissa.walsh@ouh.nhs.uk

ORCID Alissa Walsh (https://orcid.org/0000-0002-8994-1186) relationships and family life may all be disrupted, affecting self-esteem and social functioning. ${ }^{4}$

The management of chronic diseases is complex. There has been a shift from a paternalistic to a collaborative approach, which means that patients are actively involved in their own care. ${ }^{5}$ Web-based management appears to be beneficial in chronic conditions including diabetes, ${ }^{6}$ hypertension, ${ }^{7}$ and asthma. ${ }^{8,9}$ Web-based monitoring and tools improve patient confidence, communication and disease control. ${ }^{7-9}$ Involvement results in better understanding by patients of their health needs ${ }^{10}$ with the potential for clinical and economic benefit. ${ }^{11,12}$

Six web-based monitoring programs have been developed for UC. ${ }^{13-16}$ Monitoring through Home Automated Teleman- 
agement (UCHAT) showed promise during 6 months of weekly monitoring. Qualitative interviews at exit from the study showed that UCHAT resulted in increased awareness about the disease and facilitated greater control of symptoms. ${ }^{16,17} \mathrm{Be}-$
A

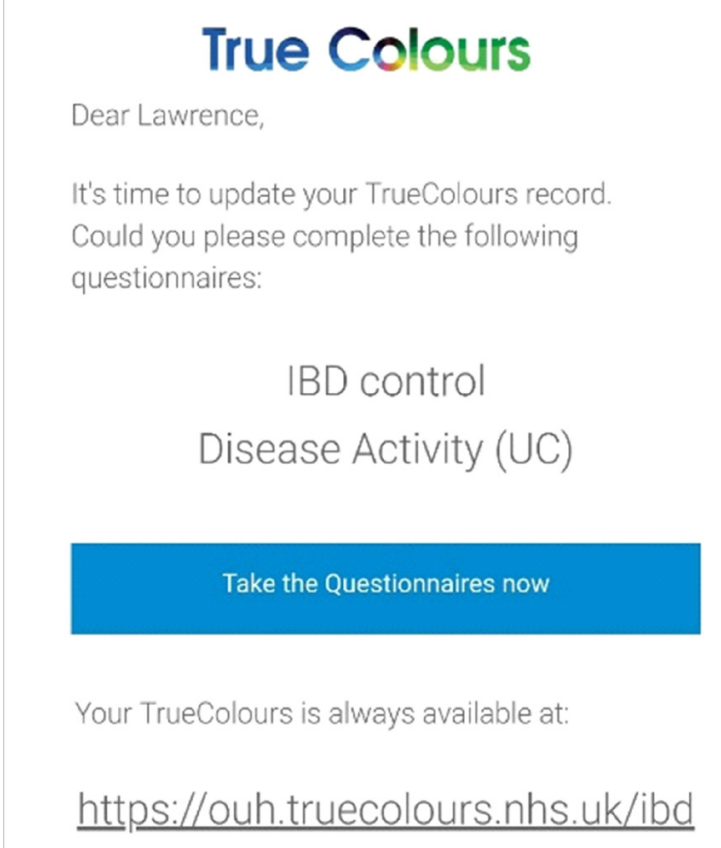

\section{B}

\section{Q1.Bowel frequency}

$0-3$

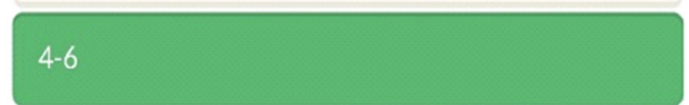

7-9

$>9$

\section{Next step $\rightarrow$}

C

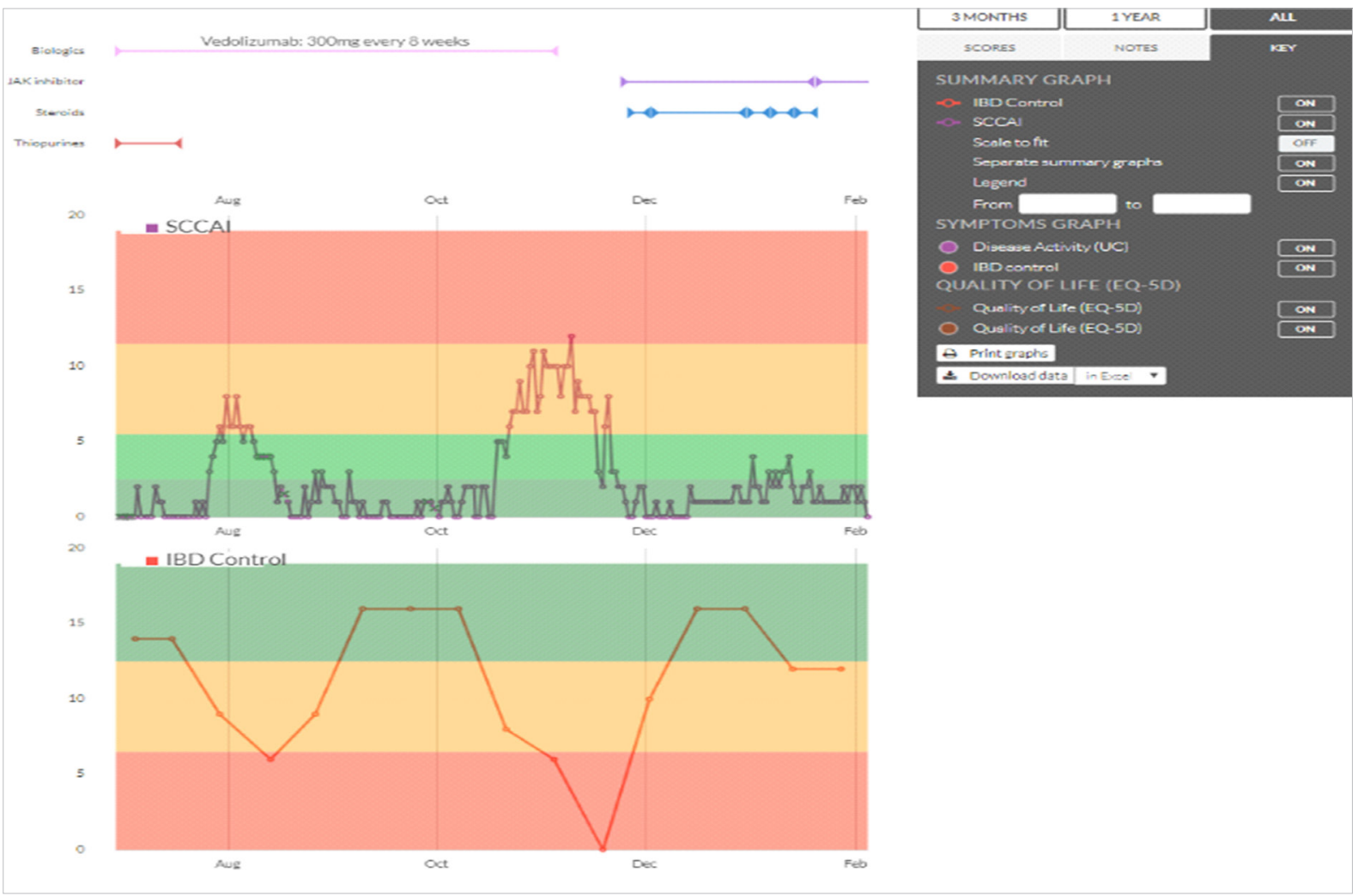

Fig. 1. Examples of TrueColours ulcerative colitis functionality. (A) Screenshot of email prompt with link to symptom and quality of life (QoL) questionnaires. (B) Screenshot of symptom questionnaire with response options. (C) Screenshot of patient dashboard displaying patient's medication timeline, SCCAI (symptoms) and IBD control (QoL) graphs. SCCAl, Simple Clinical Colitis Activity Index. 
yond this study however, there has been little published on patient perception of web-based interaction, answering questionnaires and how monitoring symptoms that fluctuate on a daily basis might affect care for UC.

TrueColours ulcerative colitis (TCUC) is a comprehensive web-based program that functions through email prompts to provide direct links to questionnaires. TCUC enables patientreported outcomes to be entered remotely (electronically through smartphone, laptop, tablet or desktop), in real-time (results immediately available) by the patient. TCUC includes a personalized dashboard that patients can visit to view their current symptom score (traffic light system of green [remission or mild symptoms], amber [moderate] and red [severe]), as well as tailored treatment guidance. The TCUC dashboard also displays graphs that allow both patient and clinician to monitor symptom and QoL trajectories along with the patient's medication timeline (Fig. 1). Outcomes agreed by the International Consortium of Health Outcome Measurement (ICHOM) for IBD, ${ }^{18}$ such as emergency department presentation or the need for hospitalization are integrated into the TCUC program.

A study was conducted to evaluate the feasibility and usability of TCUC among outpatients. In order to understand the impact of using this program from a patient perspective, qualitative interviews were conducted. Qualitative research may be unfamiliar to clinicians who are predominantly trained in quantitative measurement. The goal of qualitative research is to gain insight into people's unique experiences and their interpretation of those experiences ${ }^{19}$ and the understanding of social phenomena in natural, rather than experimental settings. ${ }^{20}$ Such insights cannot be captured by quantitative studies but they make a valuable contribution to health research.

\section{METHODS}

\section{Patients}

Patients were recruited (April 2016 to February 2017) from the Inflammatory Bowel Disease Clinic at the John Radcliffe Hospital, Oxford, UK for a 6-month pilot study. To be eligible for the TCUC pilot, patients were required to be between 18 and 65 years old, have a diagnosis of UC (European Crohn's and Colitis Organisation recommendations) ${ }_{,}^{21}$ an email address, possess a smartphone, have regular internet access and the capacity to answer questions independently. Patients were excluded if they had a diagnosis of CD or IBD unspecified, or had a colectomy.

Patients with UC were first identified by examining the elec- tronic IBD database used at the John Radcliffe Hospital, Oxford. Those who were then also part of the IBD Cohort Study, and thus consented to be contacted about other research studies, were mailed a patient invitation letter prior to their next outpatient clinic appointment. Attached to this letter was a copy of the patient information sheet, a consent form and a TCUC user guide. For those patients not sent an invitation, an invitation pack was offered during their outpatient clinic appointment. Informed consent was then obtained by one of the research team following the patient's clinical consultation. The study was approved by the Local Regional Ethical Committee (LREC reference 16/SC/0103).

\section{Patient Reported Outcomes}

A review was conducted to identify the most appropriate measures for inclusion. Only validated indices that were useful in measuring relevant outcomes were included. Symptoms were monitored using the Simple Clinical Colitis Activity Index (SCCAI).$^{22}$ The SCCAI is used in both clinical practice and clinical trials and importantly can be filled out independently, which is of utmost importance for a remote monitoring program. The SCCAI, collected daily, includes a total of 6 questions on bowel frequency, nocturnal bowel frequency, urgency of defecation, rectal bleeding, general well-being and extraintestinal manifestations (arthralgia, uveitis, skin disease), with total scores ranging from $0-2$ indicating remission, 3-5 mild disease activity, $6-11$ moderate disease activity and $\geq 12$ severe disease activities. Results were displayed using "traffic light" graphs to indicate disease activity.

Three QoL questionnaires were administered, because at the time of study design it was unclear which would be most acceptable to patients and their clinicians. The disease-specific IBD Control-8, ${ }^{23}$ subsequently selected as the QoL measure of choice by $\mathrm{ICHOM}^{18}$ includes 8 questions on patients' perceived control over their illness such as "Do you believe that your IBD has been well controlled in the past 2 weeks?", with total scores ranging from 0 to 16 indicating minimum and maximum levels of control, respectively. The other disease-specific Crohn's and Ulcerative Colitis Questionnaire- $8,{ }^{24}$ used in the CONSTRUCT trial, ${ }^{25}$ comprised 8 questions such as "In the last 2 weeks did your bowel condition prevent you from going out socially?", with total scores ranging from 0 to 8 indicating best and worst possible QoL, respectively. The generic QoL index was the EQ-5D-3L, ${ }^{26}$ comprising 5 questions on mobility, self-care, usual activities, pain/discomfort and anxiety/depression each with 3 levels-no problems, some problems and ex- 
1. What made you take part in this TrueColours ulcerative colitis program?

2. Can you describe your experience of using TrueColours?

3. Can you describe any features that you liked about using TrueColours?

4. Can you describe any features that you did not like about using TrueColours

5. Thinking about the features you liked less, what could we do to make the TrueColours system better?

6. What are the benefits or problems with using it on a regular basis?

7. How has the TrueColours ulcerative colitis program impacted on your care?

8. We used a color-coded system to graph your symptoms-green, amber and red. Do you feel that these colors accurately reflected how you felt?

9. You have been answering daily prompts for this 6-month pilot. Can you tell me how it made you feel to do this? What frequency of email prompts would you have preferred?

10. One of the aims of a technology such as this is to make a people feel more in control of their disease. What are your thoughts about this? How much do you feel that TrueColours ulcerative colitis has helped you manage your disease?

11. How would you feel about using TrueColours ulcerative colitis as part of your ongoing care?

12. Is there anything else you would like to add?

Fig. 2. Semi-structured guide for face-to-face interviews for patients using TrueColours ulcerative colitis for 6 months.

treme problems. QoL questionnaires were administered together in the above order at fortnightly intervals.

\section{Interviews}

A semi-structured interview guide was designed (A.W., S.T., and M.P.) to ask opened-ended questions for patients to describe their experience with TCUC and the impact that it had on them (Fig. 2).

To obtain a representative sample of patients from the pilot study, purposive sampling was used to invite 30 patients for interview. Audio-recorded, face-to-face interviews were conducted (A.W.), between September 2016 and January 2017, in a private room, with interview feedback being provided by M.P. Interviews occurred toward the end of the 6-month pilot to ensure that patients had had adequate exposure to TCUC. Before each interview written consent was obtained for audiorecording, and confidentiality was emphasized. Patients were encouraged to be as honest and open as possible about their experience. Interviews were anonymized and transcribed verbatim by an external professional transcription service. Transcripts were coded in NVivo 1 1, a qualitative data analysis software package. ${ }^{27}$

\section{Coding and Analysis}

Thematic analysis using an inductive approach ${ }^{28}$ was performed, with potential themes emerging from the interview transcripts during the analysis. Transcripts, which constituted the raw data were read and re-read in an iterative process to assign codes by A.W. The codes were cross-checked by S.T. and M.P. with no significant discrepancies arising. Codes relating to each theme were then grouped together. New themes that emerged as the interviews progressed were discussed and added to the existing structure and the final theme structure was agreed upon unanimously by A.W., S.T., and M.P. Saturation is widely accepted as a methodological principle in qualitative research. Data saturation is achieved when no new data emerge through further data collection. A prospective data saturation table was created to identify the point at which no new themes or insights emerged.

The Consolidated Criteria for Reporting Qualitative Research checklist was used to explicitly acknowledge that matters of transparency such as the research design and analysis being conducted by multiple investigators of varied disciplinary backgrounds. ${ }^{29}$ Patient interviews were anonymized. Each patient interviewed was randomly assigned an interview number between 1 and 28 and a master list of this allocation was documented. Patients are described by their interview number, sex (male/female $[\mathrm{M} / \mathrm{F}]$ ) and age range (years).

\section{RESULTS}

Sixty-six patients were recruited to the TCUC pilot, of which 57 (86\%) completed the 6-month pilot. Nine patients withdrew (range, day 0 to day 91$)$ due to colectomy $(n=1)$, perceived negative psychological effect $(n=1)$, inconvenience of other aspects of the TCUC pilot (e.g., additional blood tests) $(n=4)$, no reason given $(n=3)$. A total of 28 face-to-face interviews were conducted ( 2 patients declined to be interviewed). 
Table 1. Demographics of the Qualitative Cohort as Compared to the Entire Cohort

\begin{tabular}{|c|c|c|}
\hline Demographic & Qualitative cohort $(n=28)$ & Entire cohort $(n=66)$ \\
\hline Male sex ${ }^{a}$ & 46 & 44 \\
\hline Age $(y r)^{a}$ & $41.5(32.2-47.2)$ & $40.0(32.0-48.8)$ \\
\hline \multicolumn{3}{|l|}{ Worst ever extent of UC (Montreal classification) ${ }^{a, b}$} \\
\hline E1 & 14 & 21 \\
\hline E3 & 36 & 35 \\
\hline Unknown & 4 & 3 \\
\hline Tertiary education & 68 & 59 \\
\hline \multicolumn{3}{|l|}{ Smoking status } \\
\hline \multicolumn{3}{|l|}{ Prednisolone in last 12 months } \\
\hline$\leq 3$ Months & 25 & 24 \\
\hline$>3$ Months & 25 & 18 \\
\hline Hospital admissions in last 12 months $^{c}$ & 21 & 23 \\
\hline Emergency department presentations in last 12 months $^{c}$ & 18 & 20 \\
\hline Biologic use & 57 & 42 \\
\hline \multicolumn{3}{|l|}{ Disease activity (SCCAI) at entry ${ }^{a}$} \\
\hline Remission & 39 & 38 \\
\hline Time spent on internet each day (hr) & $3.0(0.0-4.0)$ & $3.0(1.0-4.5)$ \\
\hline Use of social media, such as Facebook, Twitter or Instagram & 78 & 83 \\
\hline Withdrawn patients (day of withdrawal) & $2 / 28(49,91)$ & $9 / 66(0,0,0,1,3,15,49,56,91)$ \\
\hline
\end{tabular}

Values are presented as percent or median (interquartile range).

atems used for purposive sampling.

${ }^{b}$ Montreal classification (E1: proctitis, E2: L-sided colitis, E3: extensive colitis), ${ }^{45}$ unknown $=$ answer given by patient and maximum extent unclear in medical notes.

${ }^{c}$ Median $=0$.

Two out of 28 patients interviewed had withdrawn from the study, with all other patients having completed the 6-month pilot. Demographics indicated that patients interviewed were representative of the entire cohort (Table 1). Data saturation for the qualitative interviews was reached at interview number 20 . Adherence to daily SCCAI questionnaires was $76 \%$, fortnightly QoL questionnaires 95\% and 3 months ICHOM questionnaires $100 \%$.
The 6 core themes that emerged from the analysis were: awareness, control, decision making, reassurance, communication, and burden of treatment. Not all themes were significant for all patients. There was an overarching thewme of patient empowerment which cut across all aspects of the TCUC experience. The 6 core themes are outlined with associated illustrative quotations in Table 2.

Transcending these core themes was a notion of patient 
Table 2. Quotations to Illustrate Impact of TrueColours from a Patient Perspective

\begin{tabular}{|c|c|c|}
\hline Theme & Subtheme & Interview excerpt \\
\hline \multirow[t]{2}{*}{ Awareness } & Prioritize (health) & $\begin{array}{l}\text { "...I think (it) made me more aware of it (health), instead of just brushing it to the side..." (Interview 22, } \\
\text { F 18-29 yr) }\end{array}$ \\
\hline & $\begin{array}{l}\text { Proactive disease } \\
\text { management }\end{array}$ & $\begin{array}{l}\text { "...It made me stop and think because I thought that I was OK and I clearly wasn't..." (Interview 23, } \\
\text { F 30-39 yr) }\end{array}$ \\
\hline Control & Symptom monitoring & $\begin{array}{l}\text { "...It feels like I've got more control. I've got something to physically look at and tell me, it's not in my } \\
\text { head...." (Interview 10, M 30-39 yr) }\end{array}$ \\
\hline Decision making & Medications & $\begin{array}{l}\text { "...I think it's making sure that people have the ability to have a look at their symptoms and see what's } \\
\text { working and what isn't working so that a sensible decision can be made..." (Interview 28, F 60-65 yr) }\end{array}$ \\
\hline \multirow[t]{2}{*}{ Reassurance } & $\begin{array}{l}\text { Alleviating } \\
\text { psychological } \\
\text { burden }\end{array}$ & $\begin{array}{l}\text { "...It becomes a bit more clinical to me which makes me feel better about it, because it is something that I } \\
\text { can just submit an answer to. Once the questionnaire is done I don't have to think about it..." (Interview } \\
27, \text { F 18-29 yr) }\end{array}$ \\
\hline & $\begin{array}{l}\text { Normality of } \\
\text { symptoms }\end{array}$ & $\begin{array}{l}\text { "...It was so reassuring to see that other patients must also feel anxious and tired, otherwise those questions } \\
\text { would not be there? That has helped me to feel better about these things as I haven't wanted to say } \\
\text { anything about this in my appointments..." (Interview 10, M 30-39 yr) }\end{array}$ \\
\hline \multirow[t]{4}{*}{ Communication } & $\begin{array}{l}\text { With IBD health } \\
\text { professionals }\end{array}$ & $\begin{array}{l}\text { "...I would like my dashboard to be seen at consultations as it would give a true record of my symptoms } \\
\text { and would help my specialist to see what was happening to me and what the best treatment might be..." } \\
\text { (Interview 19, F 40-49 yr) } \\
\text { "...I've definitely been one of those people who finds that you get to the appointment and you're like I've } \\
\text { been pretty good, but actually you haven't been feeling that great, so you are not really giving an accurate } \\
\text { picture..." (Interview 11, F 18-29 yr) }\end{array}$ \\
\hline & With family members & $\begin{array}{l}\text { "... Over a length of time they (my family) get a good understanding than if I did nothing and I only went for } \\
\text { my quarterly appointment...over } 6 \text { months of answering the same questions, all of this information goes } \\
\text { into your partner's mind which then feeds back with them...it's good to know that they understand...It } \\
\text { makes you talk..." (Interview 3, M 40-49 yr) }\end{array}$ \\
\hline & $\begin{array}{l}\text { Feeling connected to } \\
\text { the hospital }\end{array}$ & $\begin{array}{l}\text { "...I think the care that I have had has always been very good here but there's a psychological aspect because } \\
\text { it feels like you're connecting with the hospital every day. It feels like you are more supported..." (Interview } \\
27, \text { F 18-29 yr) }\end{array}$ \\
\hline & & $\begin{array}{l}\text { "...This whole system has made me just sort of feel like I'm getting more care from the hospital even though } \\
\text { really I'm doing the work..." (Interview 7, M 30-39 yr) }\end{array}$ \\
\hline \multirow[t]{2}{*}{$\begin{array}{l}\text { Burden of } \\
\text { treatment }\end{array}$} & On the patient & $\begin{array}{l}\text { "I'd be happy to have this monitored remotely and not come in if I was well. Obviously, there's time off work, } \\
\text { travelling to come here..." (Interview 1, M 20-29 yr) }\end{array}$ \\
\hline & $\begin{array}{l}\text { On the healthcare } \\
\text { system }\end{array}$ & $\begin{array}{l}\text { "...Anything that can alleviate pressure on the NHS and help the doctors' and the patient's time is a good } \\
\text { thing" (Interview 15, M 50-59 yr) }\end{array}$ \\
\hline
\end{tabular}

F, female; M, male; NHS, National Health Service.

empowerment which resonated above and beyond any particular theme. This was apparent in the underlying agency that patients alluded to in becoming active in their role as a patient, embracing the potential they had to self-manage and influence their disease as a result of TCUC. This concept is encapsulated by the following quotes, which suggest that TCUC may have more powerful implications for patients than any individual core theme alone could capture. The notion of TCUC promoting empowerment by allowing for a sense of progress was particularly evident among patients. Sometimes this progress was described in a psychological context.

"... Once I'd put in that I'd had a horrific night, what would have happened before TrueColours is that I'd spend the whole day thinking, "God I've had a horrific night" whereas if I had put it down, the data, I'd parked it, it had gone and I didn't think about it again because I was actually then thinking, well let's see what tomorrow brings. Psychologically I was able to look forward rather than back..." (Interview 5, F30-39yr)

In other instances, progress was alluded to in terms of patients' perceptions of their disease management and taking a leading role in this process.

"... I think that it is more about reflection. Being able to see, no I don't think I've been doing well so therefore I think I need something to change..." (Interview 11, F 18-29yr) 
Others spoke of how TCUC had instilled in them a sense of ownership of their disease, over and above a mere sense of control and an impetus to do what they could to influence their illness trajectory.

"... I think that it gives-the onus back on to me, to take some responsibility..." (Interview 28, F60-65 yr)

Finally, empowerment was demonstrated by the manner in which some patients became more assertive in their support seeking from family members as well as their healthcare team.

"... It made me feel more like I was getting the support that I needed rather than the support that they (family members) felt I needed..." (Interview 3, F 30-39 yr)

"...As soon as I got TrueColours I felt safe whereas before I had that, you know I would constantly be thinking "God am I causing problems?". I didn't want to interrupt people and the IBD nurses especially unless it was something serious. But this [TCUC] identified actually this is the time to raise your hand and say you need help..." (Interview 5, $F$ 30-39yr)

\section{DISCUSSION}

As one patient commented, "It made me stop and think": this study has shown the potential of daily digital data collection for patient empowerment. Patients reported that real-time monitoring with TCUC had a positive impact on awareness, control, decision making, reassurance, communication and had the potential to decrease their treatment burden. An overarching theme of patient empowerment permeated all these core themes.

The core themes identified by the current study and positively affected by TCUC, are those that clinical teams struggle to address when caring for patients with chronic diseases. Patients using TCUC perceived that there was improved communication on many levels: patient-IBD nurse, patient-IBD specialist and patient-relative. Of these, enhancing patient-clinician communication through the use of patient reported outcome measures (PROMs) has attracted most attention. ${ }^{30-36}$ One study ${ }^{30}$ found that completion of PROMs allowed oncology patients to express their concerns more effectively, which prompted discussion with the physician. This finding was reflected in our study with several TCUC patients stating a greater ability to express their concerns regarding symptoms such as anxiety or fatigue. Improved patient-clinician communica- tion promotes a host of benefits for the patient including improved patient satisfaction, ${ }^{37}$ which are particularly relevant to public healthcare such as the National Health Service in the United Kingdom. Patient-relative communication is also improved by PROMs in lung transplant patients, ${ }^{38}$ as we found for patients using TCUC.

Studies of mobile health monitoring in heart failure patients have shown that electronic daily self-monitoring led to feelings of increased connectedness with the hospital, reassurance and increased confidence, ${ }^{36}$ all of which were also reported when using TCUC. Another heart failure mobile monitoring program reported patient perceptions after daily measurements and symptom entry for 6 months. ${ }^{39}$ Similar to TCUC, these heart failure patients reported that they were more aware, less anxious and more confident.

It has been suggested that routine use of PROMs in chronic care management could aid shared decision making, ${ }^{40}$ as was perceived by patients using TCUC, reporting that they felt that an accurate record of their disease activity (as opposed to conventional recall) allowed patient and clinician jointly to determine whether treatment change should be considered.

Electronic monitoring was perceived by participants as having the potential to decrease clinic visits. This has been reported in heart failure telemonitoring. ${ }^{41}$ Patients in TCUC welcomed the idea of real time monitoring making better use of appointments, although patients were seen in outpatients as normal during the pilot. This emerging concept recognizes the increasing demands that are being placed on patients, acknowledging that not only do patients have the burden of their symptoms, but also a burden of treatment. ${ }^{42}$

Even though oncology, transplant, heart failure and UC are different chronic diseases, similar benefits arise from electronic self-monitoring. Ultimately, these benefits promote a sense of empowerment as outlined by our overarching theme.

Empowering patients means providing them with the opportunities and support to develop the skills, confidence and knowledge to move from being a passive recipient of care to an active partner in their health care. This enables patients to be more skilled at self-managing their condition. It changes the current healthcare paradigm from one that is predominantly prescriptive to one that is more collaborative and patient-centerd. ${ }^{10,12}$ Such self-management and empowerment has been found to support patient knowledge, behavioral change and self-efficacy, with resulting benefit on patients' overall well-being, ${ }^{10}$ as well as improved medication adherence ${ }^{43}$ which may further promote wellbeing. Patient empowerment in the cur- 
rent study, as underpinned by the 6 core themes, is supported by an extensive review into the dimensions of empowerment. ${ }^{44}$ Our analysis also unearthed dimensions of patient empowerment such as participation in decision making, gaining control, knowledge acquisition and motivation.

It is thus anticipated that TCUC will subsequently translate to better care through a number of mechanisms. For example, it is envisaged that improved disease knowledge will lead to patients engaging with their healthcare team sooner in the instance of a flare. This earlier recognition of disease exacerbation would lead to earlier treatment. Further to this, with reliable, real-time data on patients' symptoms and QoL, healthcare teams will be in a better position to interact with patients and improve care by offering the option to defer one's appointment or transfer from an outpatient clinic appointment to a nurse-led telephone clinic if the patient is demonstrably well. This will lessen the treatment burden for patients and reallocate resources for those patients in immediate need of care.

However, limitations of the current study of the current study must also be considered. Patients recruited to the TCUC pilot were self-selected and thus likely more computer literate, motivated and of a higher level of education than average. Patient activation theor ${ }^{20}$ dictates that these patients may therefore engage more effectively with platforms such as TCUC which are perceived to positively impact their health outcomes. Although this was a pilot study, these factors should be considered when calculating the impact on healthcare resources of extending such a program. It is also worth noting that while all interviews were unanimously positive in their feedback of TCUC, 5 of the 66 TCUC pilot participants did withdraw from the study due to either perceived negative psychological effect or inconvenience. As such, the TCUC platform may not be a suitable tool for all patients with UC.

Furthermore, the administration of TCUC requires trained and motivated personnel to recruit and register patients and therefore a commitment on the part of the service to provide these personnel. The most significant barrier to the success of TCUC being implemented on a wider scale is the possible resistance to it from health providers due to perceived potential disruption to workflows and increased workloads. ${ }^{45}$ Uptake of TCUC by physicians at our center has been successful as we have taken effort to educate our physicians on the benefits for both the patient and the service. We have also provided iPads within the clinic to enable physicians to readily access the patient's TCUC record.

Further research must therefore determine whether these largely positive findings from an Oxford cohort can be extrapolated to the wider UC population. Research with care providers is essential to establish whether the aforementioned proposed benefits of TCUC on workflow and workload are achievable and substantial enough to encourage long term support for use of the system. We have commenced this research at our center with TCUC being offered to all UC patients as part of routine standard of care. To date, over 500 UC patients have been registered and we await longer-term data to support use in a real-world setting.

In conclusion, patient perception of the impact of daily monitoring of UC through TCUC was extremely positive and may prove to be a way of improving management of UC while also decreasing the burden placed on both the individual and healthcare services. Moving forward, the Oxford team hope to expand TrueColours to all of their patients with UC, as well as expanding the program to incorporate $\mathrm{CD}$.

\section{FINANCIAL SUPPORT}

This work was supported by the National Institute for Health Research (NIHR) Oxford Biomedical Research Centre (BRC).

\section{CONFLICT OF INTEREST}

The TrueColours ulcerative colitis pilot was funded by the Norman Collisson Foundation, Buhlmann Laboratories, AbbVie Pharmaceuticals and Takeda. However, all of these are not relevant to this article.

\section{AUTHOR CONTRIBUTION}

Conception and design of the study: Walsh A, Hinds C, Geddes J, Goodwin G, Collins G, Peters M. Design of the study, revising the article: Brain O, Keshav S. Acquisition of data: Walsh A, Hinds C, Sexton V, Geddes J, Goodwin G, Travis S, Peters M. Analysis and interpretation of data: Walsh A, Matini L, Collins G, Travis S, Peters M. Drafting the article: Walsh A, Matini L, Collins G, Travis T, Peters M. Approval of final manuscript: all authors.

\section{ACKNOWLEDGEMENTS}

We thank all patients for participation in this study and the Research Nurses of the Translational Gastroenterology Unit and Weatherall Institute of Molecular Medicine in particular, 
supported in part by the NIHR Local Clinical Research Network. We acknowledge the contribution of the BRC Gastrointestinal biobank, which is supported by the NIHR Oxford Biomedical Research Centre (11/YH/0020, 16/YH/0247). We acknowledge the contribution of Oxford IBD Cohort Investigators: Carolina Arancibia, Adam Bailey, Ellie Barnes, Beth BirdLieberman, Oliver Brain, Barbara Braden, Jane Collier, James East, Alessandra Geremia, Lucy Howarth, Paul Klenerman, Simon Leedham, Rebecca Palmer, Fiona Powrie, Astor Rodrigues, Alison Simmons, Peter Sullivan. We acknowledge the collaboration of Satish Keshav who sadly died during the revision process of this manuscript and who was instrumental to setup the Oxford IBD cohort study.

\section{REFERENCES}

1. Cosnes J, Gower-Rousseau C, Seksik P, Cortot A. Epidemiology and natural history of inflammatory bowel diseases. Gastroenterology 2011;140:1785-1794.

2. Moradkhani A, Beckman LJ, Tabibian JH. Health-related quality of life in inflammatory bowel disease: psychosocial, clinical, socioeconomic, and demographic predictors. J Crohns Colitis 2013;7:467-473.

3. Irvine EJ. Quality of life of patients with ulcerative colitis: past, present, and future. Inflamm Bowel Dis 2008;14:554-565.

4. Ghosh S, Mitchell R. Impact of inflammatory bowel disease on quality of life: results of the European Federation of Crohn's and Ulcerative Colitis Associations (EFCCA) patient survey. J Crohns Colitis 2007;1:10-20.

5. Chiauzzi E, Rodarte C, DasMahapatra P. Patient-centered activity monitoring in the self-management of chronic health conditions. BMC Med 2015;13:77.

6. Franklin V, Waller A, Pagliari C, Greene S. "Sweet Talk": text messaging support for intensive insulin therapy for young people with diabetes. Diabetes Technol Ther 2003;5:991-996.

7. Friedman RH, Kazis LE, Jette A, et al. A telecommunications system for monitoring and counseling patients with hypertension. Impact on medication adherence and blood pressure control. Am J Hypertens 1996;9:285-292.

8. Pinnock H, Slack R, Pagliari C, Price D, Sheikh A. Professional and patient attitudes to using mobile phone technology to monitor asthma: questionnaire survey. Prim Care Respir J 2006;15:237-245

9. Rasmussen LM, Phanareth K, Nolte H, Backer V. Internet-based monitoring of asthma: a long-term, randomized clinical study of 300 asthmatic subjects. J Allergy Clin Immunol 2005;115:
$1137-1142$

10. Barlow J, Wright C, Sheasby J, Turner A, Hainsworth J. Selfmanagement approaches for people with chronic conditions: a review. Patient Educ Couns 2002;48:177-187.

11. Dinesen B, Andersen PE. Qualitative evaluation of a diabetes advisory system, DiasNet. J Telemed Telecare 2006;12:71-74.

12. Dinesen B, Nøhr C, Andersen SK, Sejersen H, Toft E. Under surveillance, yet looked after: telehomecare as viewed by patients and their spouse/partners. Eur J Cardiovasc Nurs 2008; 7:239-246.

13. Atreja A, Khan S, Rogers JD, et al. Impact of the mobile HealthPROMISE platform on the quality of care and quality of life in patients with inflammatory bowel disease: study protocol of a pragmatic randomized controlled trial. JMIR Res Protoc 2015; 4:e23.

14. de Jong M, van der Meulen-de Jong A, Romberg-Camps M, et al. Development and feasibility study of a telemedicine tool for all patients with IBD: MyIBDcoach. Inflamm Bowel Dis 2017;23:485-493

15. Elkjaer M, Shuhaibar M, Burisch J, et al. E-health empowers patients with ulcerative colitis: a randomised controlled trial of the web-guided 'Constant-care’ approach. Gut 2010;59:16521661.

16. Cross RK, Arora M, Finkelstein J. Acceptance of telemanagement is high in patients with inflammatory bowel disease. J Clin Gastroenterol 2006;40:200-208.

17. Castro HK, Cross RK, Finkelstein J. Using a Home Automated Telemanagement (HAT) system: experiences and perceptions of patients with inflammatory bowel disease. AMIA Annu Symp Proc 2006;2006:872.

18. Kim AH, Roberts C, Feagan BG, et al. Developing a standard set of patient-centred outcomes for inflammatory bowel disease-an international, cross-disciplinary consensus. J Crohns Colitis 2018;12:408-418.

19. Peters M, Abu-Saad HH, Vydelingum V, Murphy M. Research into headache: the contribution of qualitative methods. Headache 2002;42:1051-1059.

20. Pope C, Mays N. Reaching the parts other methods cannot reach: an introduction to qualitative methods in health and health services research. BMJ 1995;311:42-45.

21. Magro F, Gionchetti P, Eliakim R, et al. Third European evidence-based consensus on diagnosis and management of ulcerative colitis. Part 1: definitions, diagnosis, extra-intestinal manifestations, pregnancy, cancer surveillance, surgery, and ileo-anal pouch disorders. J Crohns Colitis 2017;11:649-670.

22. Walmsley RS, Ayres RC, Pounder RE, Allan RN. A Simple Clini- 
cal Colitis Activity Index. Gut 1998;43:29-32.

23. Bodger K, Ormerod C, Shackcloth D, Harrison M; IBD Control Collaborative. Development and validation of a rapid, generic measure of disease control from the patient's perspective: the IBD-control questionnaire. Gut 2014;63:1092-1102.

24. Alrubaiy L, Cheung WY, Dodds P, et al. Development of a short questionnaire to assess the quality of life in Crohn's disease and ulcerative colitis. J Crohns Colitis 2015;9:66-76.

25. Williams JG, Alam MF, Alrubaiy L, et al. Comparison Of iNfliximab and ciclosporin in STeroid Resistant Ulcerative Colitis: pragmatic randomised Trial and economic evaluation (CONSTRUCT). Health Technol Assess 2016;20:1-320.

26. EuroQol Group. EuroQol: a new facility for the measurement of health-related quality of life. Health Policy 1990;16:199-208.

27. NVivo qualitative data analysis software, version 11. QSR International Pty Ltd: Melbourne, 2015.

28. Braun V, Clarke V. Using thematic analysis in psychology. Qual Res Psychol 2006;3:77-101.

29. Tong A, Sainsbury P, Craig J. Consolidated Criteria for Reporting Qualitative Research (COREQ): a 32-item checklist for interviews and focus groups. Int J Qual Health Care 2007;19:349357.

30. Velikova G, Booth L, Smith AB, et al. Measuring quality of life in routine oncology practice improves communication and patient well-being: a randomized controlled trial. J Clin Oncol 2004;22:714-724.

31. Detmar SB, Muller MJ, Schornagel JH, Wever LD, Aaronson NK. Health-related quality-of-life assessments and patientphysician communication: a randomized controlled trial. JAMA 2002;288:3027-3034.

32. Haywood K, Marshall S, Fitzpatrick R. Patient participation in the consultation process: a structured review of intervention strategies. Patient Educ Couns 2006;63:12-23.

33. Gerrity P. And to think that it happened on 11th street: a nursing approach to community-based holistic care and health care reform. Altern Ther Health Med 2010;16:62-67.

34. Takeuchi EE, Keding A, Awad N, et al. Impact of patient-reported outcomes in oncology: a longitudinal analysis of patient-physician communication. J Clin Oncol 2011;29:29102917.
35. Harley C, Takeuchi E, Taylor S, et al. A mixed methods approach to adapting health-related quality of life measures for use in routine oncology clinical practice. Qual Life Res 2012;21: 389-403.

36. Chantler T, Paton C, Velardo C, et al. Creating connections the development of a mobile-health monitoring system for heart failure: qualitative findings from a usability cohort study. Digit Health 2016;2:2055207616671461.

37. Ha JF, Longnecker N. Doctor-patient communication: a review. Ochsner J 2010;10:38-43.

38. Santana MJ, Feeny D, Johnson JA, et al. Assessing the use of health-related quality of life measures in the routine clinical care of lung-transplant patients. Qual Life Res 2010;19:371379.

39. Seto E, Leonard KJ, Cafazzo JA, Barnsley J, Masino C, Ross HJ. Perceptions and experiences of heart failure patients and clinicians on the use of mobile phone-based telemonitoring. J Med Internet Res 2012;14:e25.

40. Santana MJ, Feeny D. Framework to assess the effects of using patient-reported outcome measures in chronic care management. Qual Life Res 2014;23:1505-1513.

41. Seto E, Leonard KJ, Cafazzo JA, Barnsley J, Masino C, Ross HJ. Mobile phone-based telemonitoring for heart failure management: a randomized controlled trial. J Med Internet Res 2012; 14:e31.

42. Eton DT, Yost KJ, Lai JS, et al. Development and validation of the Patient Experience with Treatment and Self-management (PETS): a patient-reported measure of treatment burden. Qual Life Res 2017;26:489-503.

43. Hernandez-Tejada MA, Campbell JA, Walker RJ, Smalls BL, Davis KS, Egede LE. Diabetes empowerment, medication adherence and self-care behaviors in adults with type 2 diabetes. Diabetes Technol Ther 2012;14:630-634.

44. Cerezo PG, Juvé-Udina ME, Delgado-Hito P. Concepts and measures of patient empowerment: a comprehensive review. Rev Esc Enferm USP 2016;50:667-674.

45. Satsangi J, Silverberg MS, Vermeire S, Colombel JF. The Montreal classification of inflammatory bowel disease: controversies, consensus, and implications. Gut 2006;55:749-753. 